\title{
Analysis of polarimetric parameters in strongly oriented biological tissues
}

\author{
F. Fanjul-Vélez, J. L. Arce-Diego \\ Applied Optical Techniques Group, TEISA Department, University of Cantabria, Av de los Castros s/n, 39005 Santander (Spain) \\ fanjulf@unican.es; arcedj@unican.es
}

\begin{abstract}
The use of polarimetry as a diagnostic tool for biological tissues could show increased contrast in several pathologies. In this work polarimetric parameters are used to analyze structurally oriented biological tissues.

OCIS codes: (260.5430) Polarization; (110.5405) Polarimetric imaging; (170.4580) Optical diagnostics for medicine; (260.1440) Birefringence
\end{abstract}

\section{Introduction}

The use of optical techniques for tissue diagnosis provides non-invasive, non-contact, high resolution and increased contrast. In particular, the use of the polarization information can add contrast, particularly in strongly oriented biological tissues. Most of these tissues exhibit strong birefringence, particularly when they are collagen-based, like tendons, ligaments or cartilage [1]. The study of these tissues can be implemented by polarization sensitive techniques, such as Polarized Light Microscopy (PLM) or Polarization Sensitive Optical Coherence Tomography (PS-OCT) [2].

Among the most significant polarimetric parameters of strongly oriented biological tissues are birefringence and optic axis orientation. In order to analyze these or other polarimetric parameters, the measurement of Mueller matrix contains all the polarimetric information of the sample [3]. The expected polarimetric response can be estimated by extended Jones calculus [4], considering that the incident radiation could be not perpendicular to the tissue and/or the fibril orientation of the collagen inside is arbitrary, like in Full-Field PS-OCT on curved structures. Afterwards Muller matrix can be measured by means of a full Mueller polarimeter. With these results several decompositions can calculations can be made, such as the Lu Chipman decomposition [5], the Mueller Coherency matrix [6] or the differential Jones matrix [7].

In this work strongly oriented biological tissues, such as muscular and nerve tissues, are analysed by a Mueller matrix system. The system is based on liquid crystal variable retarders, and provides the 16 elements of the Mueller matrix. Ex vivo pig tissues are modeled and measured.

\section{Methods}

Biological tissues are considered as strongly oriented retarders, with particular fibrils orientation and birefringence. The employed model is based on the electromagnetic propagation of light in birefringent media. The tissue is modeled as a positive uniaxial crystal whose optic axis is coincident with the fibrils direction. Figure 1 shows an scheme with the main axes and parameters, including the extraordinary and ordinary refractive indexes, and the orientation of the optic axis c. Propagation is modeled by vector $\mathrm{k}$ for the incident light in an isotropic medium and vectors $\mathrm{k}_{0}$ and $\mathrm{k}_{\mathrm{e}}$ for the birefringent medium. The incident and output Jones vectors are referred to a system defined by TE and TM incident vectors s and p, respectively. Dichroism, optical activity and multiple reflections in the inner interfaces are neglected. The collagen-based birefringent $\mathrm{N}$-layered biological tissue is described by the product of $2 \times 2$ Extended Jones matrices:

$$
\begin{aligned}
& J_{\text {out }}=M_{T} J_{i n}=D_{f i n} P_{N} T_{N-1, N} P_{N-1} T_{N-2, N-1} \ldots T_{2,3} P_{2} T_{1,2} P_{1} D_{i n i} J_{i n}= \\
& =\left(\begin{array}{cc}
\mathbf{o} \cdot \mathbf{s} t_{s}^{\prime} & \mathbf{s} \cdot \mathbf{e} t_{s}^{\prime} \\
\mathbf{p}_{o} \cdot \mathbf{o} t_{p}^{\prime} & \mathbf{p}_{o} \cdot \mathbf{e} t_{p}^{\prime}
\end{array}\right)\left(\begin{array}{cc}
e^{-j k_{o z} d_{N}} & 0 \\
0 & e^{-j k_{e z} d_{N}}
\end{array}\right)\left(\begin{array}{cc}
\mathbf{o}_{N-1} \mathbf{o}_{N} & \mathbf{e}_{N-1} \cdot \mathbf{o}_{N} \\
\mathbf{o}_{N-1} \cdot \mathbf{e}_{N} & \mathbf{e}_{N-1} \cdot \mathbf{e}_{N}
\end{array}\right) \cdots\left(\begin{array}{cc}
\mathbf{s} \cdot \mathbf{o} t_{s} & \mathbf{p}_{o} \cdot \mathbf{o} t_{p} \\
\mathbf{s} \cdot \mathbf{e} t_{s} & \mathbf{p}_{o} \cdot \mathbf{e} t_{p}
\end{array}\right) J_{i n}
\end{aligned}
$$

$J_{\text {in }}$ is the incident Jones vector; $J_{\text {out }}$ the output Jones vector; $M_{T}$ is the Extended Jones matrix of the biological tissue; matrices $D_{i n i}$ and $D_{\text {fin }}$ model the interaction at the interface between an isotropic medium (usually air) and the biological tissue; $P_{i}$ are propagation matrices through the $i$ th layer, of depth $d_{i} ; T_{i, i+1}$ are the dynamical propagation 
matrices between uniaxial layers $i$ and $i+1 ; \mathbf{0}$ and $\mathbf{e}$ are the ordinary and extraordinary polarization vectors; $\mathbf{p}_{\mathbf{o}}=\mathbf{k}_{\mathbf{0}} \mathbf{x} \mathbf{s}$; and $t_{s}, t_{p}, t_{s}^{\prime}, t_{p}^{\prime}$ are the Fresnel transmission coefficients.

Mueller matrix polarimeter is based on four liquid crystal variable retarders (Meadowlarks Inc.), in a reflection configuration. The system is calibrated by the eigenvalues calibration method [8]. The optical source is a white light broadband QTH source (Newport Inc.), which is previously filtered. A diagram is shown in Figure 1.

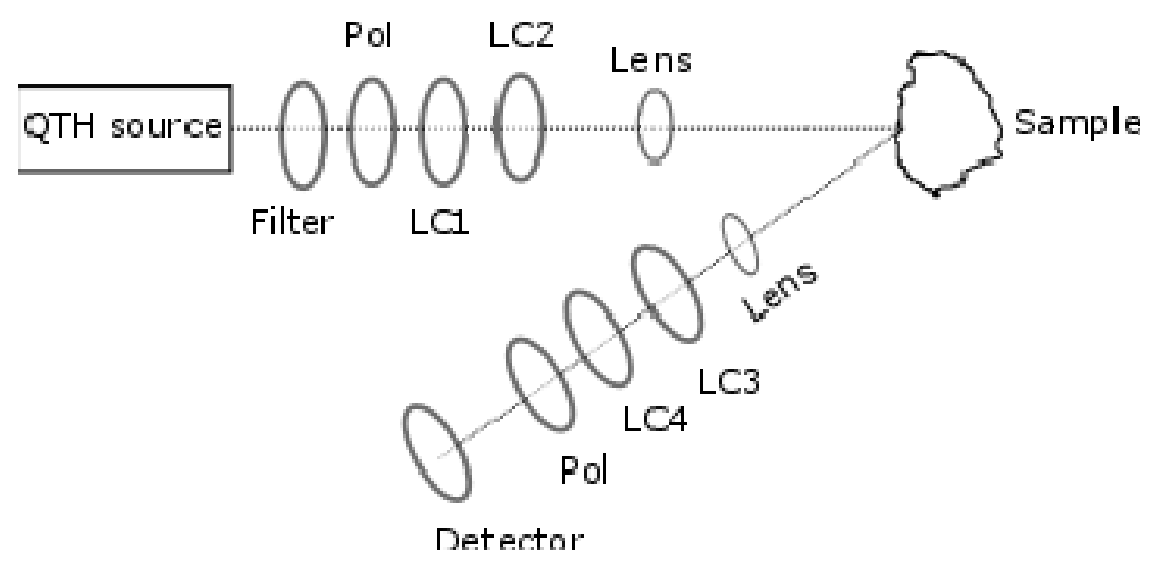

Fig 1. Diagram of the Mueller polarimeter: Pol: linear polarizer; LC: Liquid crystal variable retarder.

Matrix analysis is mainly achieved by the comparison with the estimated global response obtained by the previous method. Afterwards Lu Chipman decomposition is applied, the diattenuation, retardance and depolarization matrices are obtained:

$$
\begin{gathered}
M=M_{\Delta} M_{R} M_{D} \\
M_{D}=m_{00}\left(\begin{array}{cc}
1 & D^{t} \\
D & m_{D}
\end{array}\right) \\
m_{\Delta}=\varepsilon\left[m^{\prime}\left(m^{\prime}\right)^{t}+\left(\sqrt{\lambda_{1} \lambda_{2}}+\sqrt{\lambda_{2} \lambda_{3}}+\sqrt{\lambda_{1} \lambda_{3}}\right) I\right]^{-1} \cdot\left[\left(\sqrt{\lambda_{1}}+\sqrt{\lambda_{2}}+\sqrt{\lambda_{3}}\right) m^{\prime}\left(m^{\prime}\right)^{t}+\sqrt{\lambda_{1} \lambda_{2} \lambda_{3}} I\right] \\
M_{\Delta}=\left[\begin{array}{ll}
1 & \overrightarrow{0}^{t} \\
\vec{P}_{\Delta} & \mathrm{m}_{\Delta}
\end{array}\right]
\end{gathered}
$$

Mueller Coherency matrix method is also considered, from Pauli matrices $\sigma_{i}$ :

$$
\left.\begin{array}{l}
\boldsymbol{\rho}_{i}=\boldsymbol{\sigma}_{0} \otimes \boldsymbol{\sigma}_{i} \\
\boldsymbol{\rho}_{i}^{\prime}=\boldsymbol{\sigma}_{i} \otimes \boldsymbol{\sigma}_{0}
\end{array}\right\} \Rightarrow \boldsymbol{\eta}_{4 i+j}=\boldsymbol{\rho}_{i}^{\prime} \cdot \boldsymbol{\rho}_{j}
$$

The use of the Dirac matrices allows the connection of the Mueller matrix elements $m_{i j}$ with a $4 \times 4$ coherence matrix, $\mathbf{C}_{\mathbf{4} \mathbf{x} 4}$, which is directly related with the properties of the optical device. This $4 \times 4$ coherence matrix is defined from the $S U(4) \leftrightarrow O^{+}(6)$ homomorphism by the Mueller matrix elements and Dirac matrices as follows: 


$$
\begin{aligned}
& \mathbf{C}_{4 \mathbf{x} 4}=\frac{1}{4} \sum_{i, j=0}^{3} m_{i j} \eta_{i j} \\
& \boldsymbol{\eta}_{0}=\left[\begin{array}{cccc}
1 & 0 & 0 & 0 \\
0 & 1 & 0 & 0 \\
0 & 0 & 1 & 0 \\
0 & 0 & 0 & 1
\end{array}\right] \quad \boldsymbol{\eta}_{1}=\left[\begin{array}{cccc}
0 & 1 & 0 & 0 \\
1 & 0 & 0 & 0 \\
0 & 0 & 0 & i \\
0 & 0 & -i & 0
\end{array}\right] \quad \boldsymbol{\eta}_{2}=\left[\begin{array}{cccc}
0 & 0 & 1 & 0 \\
0 & 0 & 0 & -i \\
1 & 0 & 0 & 0 \\
0 & i & 0 & 0
\end{array}\right] \quad \boldsymbol{\eta}_{3}=\left[\begin{array}{cccc}
0 & 0 & 0 & 1 \\
0 & 0 & i & 0 \\
0 & -i & 0 & 0 \\
1 & 0 & 0 & 0
\end{array}\right] \\
& \boldsymbol{\eta}_{4}=\left[\begin{array}{cccc}
0 & 1 & 0 & 0 \\
1 & 0 & 0 & 0 \\
0 & 0 & 0 & -i \\
0 & 0 & i & 0
\end{array}\right] \quad \boldsymbol{\eta}_{5}=\left[\begin{array}{cccc}
1 & 0 & 0 & 0 \\
0 & 1 & 0 & 0 \\
0 & 0 & -1 & 0 \\
0 & 0 & 0 & -1
\end{array}\right] \quad \boldsymbol{\eta}_{6}=\left[\begin{array}{cccc}
0 & 0 & 0 & -i \\
0 & 0 & 1 & 0 \\
0 & 1 & 0 & 0 \\
i & 0 & 0 & 0
\end{array}\right] \quad \boldsymbol{\eta}_{7}=\left[\begin{array}{cccc}
0 & 0 & 1 & 0 \\
0 & 0 & 0 & -i \\
1 & 0 & 0 & 0 \\
0 & i & 0 & 0
\end{array}\right] \\
& \boldsymbol{\eta}_{8}=\left[\begin{array}{cccc}
0 & 0 & 1 & 0 \\
0 & 0 & 0 & i \\
1 & 0 & 0 & 0 \\
0 & -i & 0 & 0
\end{array}\right] \quad \boldsymbol{\eta}_{9}=\left[\begin{array}{cccc}
0 & 0 & 0 & i \\
0 & 0 & 1 & 0 \\
0 & 1 & 0 & 0 \\
-i & 0 & 0 & 0
\end{array}\right] \quad \boldsymbol{\eta}_{\mathbf{1 0}}=\left[\begin{array}{cccc}
1 & 0 & 0 & 0 \\
0 & -1 & 0 & 0 \\
0 & 0 & 1 & 0 \\
0 & 0 & 0 & -1
\end{array}\right] \quad \boldsymbol{\eta}_{\mathbf{1 1}}=\left[\begin{array}{cccc}
0 & -i & 0 & 0 \\
i & 0 & 0 & 0 \\
0 & 0 & 0 & 1 \\
0 & 0 & 1 & 0
\end{array}\right] \\
& \boldsymbol{\eta}_{\mathbf{1 2}}=\left[\begin{array}{cccc}
0 & 0 & 0 & 1 \\
0 & 0 & -i & 0 \\
0 & i & 0 & 0 \\
1 & 0 & 0 & 0
\end{array}\right] \quad \boldsymbol{\eta}_{13}=\left[\begin{array}{cccc}
0 & 0 & -i & 0 \\
0 & 0 & 0 & 1 \\
i & 0 & 0 & 0 \\
0 & 1 & 0 & 0
\end{array}\right] \quad \boldsymbol{\eta}_{14}=\left[\begin{array}{cccc}
0 & i & 0 & 0 \\
-i & 0 & 0 & 0 \\
0 & 0 & 0 & 1 \\
0 & 0 & 1 & 0
\end{array}\right] \quad \boldsymbol{\eta}_{15}=\left[\begin{array}{cccc}
1 & 0 & 0 & 0 \\
0 & -1 & 0 & 0 \\
0 & 0 & -1 & 0 \\
0 & 0 & 0 & 1
\end{array}\right] \\
& \mathbf{C}_{4 \mathbf{x} 4}=\lambda_{1} \mathbf{C}_{1}+\lambda_{2} \mathbf{C}_{2}+\lambda_{3} \mathbf{C}_{3}+\lambda_{4} \mathbf{C}_{4}
\end{aligned}
$$

The number of significant (non-zero) eigenvalues and their values are directly related to the depolarizing characteristics of the optical media. The entropy-factor $H$ is then defined as:

$$
H=-\sum x_{i} \log _{4}\left(x_{i}\right) \rightarrow x_{i}=\frac{\lambda_{i}}{\sum_{j} \lambda_{j}}
$$

\section{Results and discussion}

Ex vivo pig biological tissues of muscle and verve tissues are employed. Their real orientation is visually and microscopically estimated. All of them are modeled according to the previous section methods, and measured with the Mueller matrix polarimeter. Figure 2 show one of the samples, and Figure 3 shows an example of entropy map. 


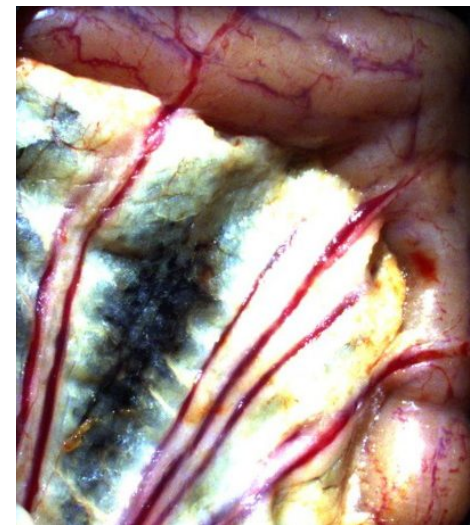

Fig 2. Ex-vivo pig biological tissue sample.

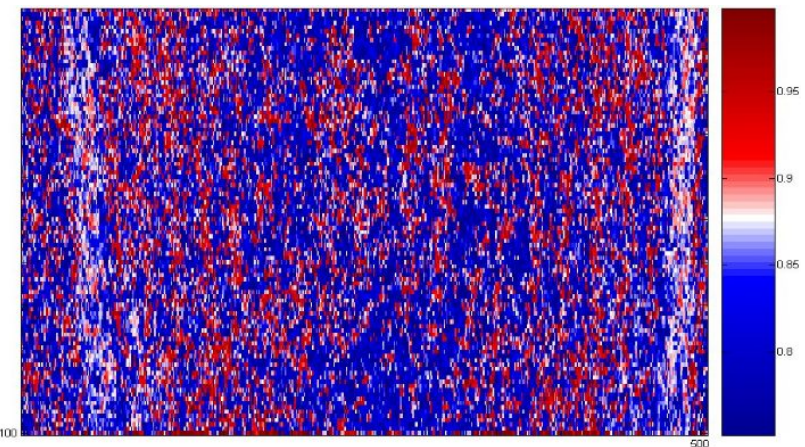

Fig 3. Entropy map of one of the samples.

The results obtained by means of the decomposition and analysis methods provide different estimations of the preferred orientation of the tissues. The estimation by the model further contributes to suppress experimental uncertainty. The adequate consideration of tissue orientation is of great relevance for several pathologies, particularly for corneal diseases, such as keratoconus, or articular disorders, such as those of the hyaline cartilage.

\section{Acknowledgements}

This work has been partially supported by the project "New active phases in transition metals and rare earth nano-oxides stabilized at high pressure" (MAT2015-69508-P) of the Spanish Ministry of Economy and Competitiveness, cofunded by FEDER funds, and by the San Cándido Foundation.

\section{References}

[1] Fratzl, P., [Collagen: Structure and Mechanics], Springer (2008).

[2] Fanjul-Vélez, F., Pircher, M., Baumann, B., Götzinger, E., Hitzenberger, C. K. and Arce-Diego, J. L., "Polarimetric analysis of the human cornea by Polarization Sensitive Optical Coherence Tomography,” Journal of Biomedical Optics 15, 056004 (2010).

[3] Fanjul-Vélez, F., Ortega-Quijano, N. and Arce-Diego, J. L., "Polarimetry Group Theory analysis in biological tissue phantoms by Mueller Coherency matrix," Optics Communications 283, 4525-4530 (2010).

[4] Fanjul-Vélez, F. and, Arce-Diego, J. L., "Polarimetry of birefringent biological tissues with arbitrary fibril orientation and variable incidence angle," Optics Letters 35, 1163-1165 (2010).

[5] Lu, S.Y. and Chipman, R.A., "Interpretation of Mueller matrices based on polar decomposition," JOSA A 13, 1106-1113 (1996). 
[6] Fanjul-Vélez, F., Ortega-Quijano, N. and Arce-Diego, J. L., "Polarimetry Group Theory analysis in biological tissue phantoms by Mueller Coherency matrix," Optics Communications 283, 4525-4530 (2010).

[7] Ortega-Quijano, N., Fanjul-Vélez, F. and Arce-Diego, J.L., "Polarimetric study of birefringent turbid media with three-dimensional optic axis orientation," Biomedical Optics Express 5, 287-292 (2014).

[8] De Martino, A., Kim, Y.K., Garcia-Caurel, E., Laude, B. and Drévillon, B., “Optimized Mueller polarimeter with liquid crystals,” Optics Letters 28, 616-618 (2003). 